\title{
Does the number of prior oocyte retrieval practices increase the amount of blood loss in subsequent OPU procedures?
}

\author{
Özkan Özdamar ${ }^{1 *}$, İsmet Gün², Ali Ovayolu ${ }^{2}$, Kenan Sofuoğlu ${ }^{2}$
}

\author{
${ }^{1}$ Department of Obstetrics and Gynecology, Gölcük Military Hospital, Kocaeli, Turkey, \\ ${ }^{2}$ Department of Obstetrics and Gynecology, Zeynep Kamil Maternity and Children's Training and Educational \\ Hospital, Istanbul, Turkey
}

Received: 14 October 2015

Accepted: 23 November 2015

\author{
*Correspondence: \\ Dr. Özkan Özdamar, \\ E-mail: ozkan_ozdamar35@hotmail.com
}

Copyright: $\odot$ the author(s), publisher and licensee Medip Academy. This is an open-access article distributed under the terms of the Creative Commons Attribution Non-Commercial License, which permits unrestricted non-commercial use, distribution, and reproduction in any medium, provided the original work is properly cited.

\begin{abstract}
Background: Though OPU is a safe and simple procedure, it may cause damage to pelvic structures and bleeding. It is unclear whether history of a prior OPU poses any risk of increased blood loss in the OPU. We, herein, aimed to evaluate whether mean blood loss and hematologic parameters vary between women with and without the history of an OPU, in an unexplained infertility population.

Methods: A prospective case-control study was conducted through the files of patients who underwent IVF/ICSI-ET between June and December 2013. Unexplained infertility patients with a CBC result were assigned to two groups; those undergoing OPU for the first time (Group 1, n=40) and those who were previously subjected to at least 1 procedure (Group 2, $\mathrm{n}=13$ ). Blood samples were obtained before the ET. Hemoglobin values and changes were compared between the two groups.

Results: Delta hemoglobin value did not significantly differ between the groups $(\mathrm{p}=0.469)$ and there were no significant difference between the pre- and post-procedure hemoglobin values in the patients with no and at least one OPUs and the total patient population ( $\mathrm{p}=0.792,0.259$ and 0.442 , respectively).

Conclusions: Our study results reveal that the amount of the blood loss in OPU does not significantly vary between women subjected to the procedure for the first time and for more than 1 time.
\end{abstract}

Keywords: Oocyte retrieval, In-vitro fertilization, Hemorrhage, Hemoglobin

\section{INTRODUCTION}

Transvaginal ultrasound-guided follicle aspiration has become the gold-standard procedure for oocyte retrieval in in-vitro fertilization (IVF) and intracytoplasmic sperm injection (ICSI) over the past two decades. ${ }^{1}$ The safety, simplicity, low costs and ease in the learning of the procedure in addition to minimal discomfort and quick postoperative recovery for the patients have rendered it more popular and more adopted than the previous oocyte retrieval techniques. ${ }^{2}$ Nevertheless, the procedure can cause damage to vaginal mucosa, ovaries, adjacent pelvic organs or blood vessels traumatized by the aspiration needle, which may result in internal or external bleeding, varying from mild to severe, or other complications. ${ }^{3,4}$ Clinically significant bleeding after OPU is uncommon with a reported incidence of severe intra- or retroperitoneal bleeding varying between 0 and $1.3 \%$ and is reported to be clinically unremarkable in the vast majority of women undergoing OPU. ${ }^{5}$ The pooling data suggests that in patients with coagulation abnormalities including von Willebrand disease (VWD), ${ }^{6}$ thrombocythemia, ${ }^{7}$ the risk of post-procedure bleeding is higher than the general population. However, the risk 
factors for clinically significant blood loss after OPU have not been extensively studied and, to the best to our knowledge, data regarding the risk factors, other than coagulopathies, for the post-procedure bleeding is lacking.

Now that the clinical pregnancy rate per embryo transferred is still modest, ${ }^{8}$ a significant proportion of couples undergoing IVF/ICSI-ET cycles would only benefit from recurrent ART attempts, which, in turn, result in recurrent oocyte retrieval procedures. In this context, it is unclear whether, or not, history of a prior OPU, similar to the history of a surgery, ${ }^{9}$ pose any risk of increased blood loss in the course of OPU. We, herein, aimed to determine whether mean blood loss and hematologic parameters vary between women with and without the history of an oocyte retrieval procedure, in a non-anemic, unexplained infertility population who underwent OPU under general anesthesia, following controlled ovarian stimulation (COS).

\section{METHODS}

This study has been performed in accordance with the ethical standards described in the 1975 declaration of Helsinki, as revised in 2000. The present is a prospective case-control study conducted through the files of patients who admitted to the assisted reproduction department of Zeynep Kamil training and educational hospital due to the desire of having a child and who were agreed to undergo IVF/ICSI treatments. Since the present is an assessment of conventionally treated patients, no intervention was involved beyond routine and standard arrangement and treatment and no patient-identifying information was included, the Institutional Review Board waived the requirements for approval of this study and patient consent. All couples undergoing IVF or ICSI treatment between June and December 2013 were considered for enrolment in the trial. Only the patients whose complete blood count samples had been obtained as a part of infertility work-up prior to both OPU and ET were included for assessment. Inclusion criteria were as follows; age between 23-39 years and body mass index $(\mathrm{BMI}) \leq 28 \mathrm{~kg} / \mathrm{m}^{2}, \mathrm{FSH} \leq 10 \mathrm{mIU} / \mathrm{mL}$ and unexplained infertility patients who underwent either agonist or antagonist protocol. The exclusion criteria included presence of coagulopathies such as VWD, thrombocythemia or hemophilia, previous abdominal or vaginal surgery and any kind of ovarian or adnexal pathologies. Besides, patients who developed ovarian hyperstimulation syndrome (OHSS) in the course of controlled ovarian stimulation were excluded from the study, in order to avoid any potential biases on the results that would have arisen from hemoconcentration. With a similar rationale, patients who were potential candidates to highly respond to COS and to develop OHSS, i.e. those with a previous history of OHSS, polycystic ovary syndrome (PCOS) or an AMH level >4.6 ng/mL, were excluded.
Either a classical long agonist or fixed and/or flexible antagonist protocol was applied for ovarian stimulation. The treatment protocol choices and the gonadotropin dose adjustments were individualized according to age, body mass index (BMI), ovarian reserve determined by antral follicle count and basal $\mathrm{FSH}$, and experience from previous cycles. Gonadotropin stimulation was achieved by administering either rFSH or HMG. All patients were given folic acid 400 mcg daily simultaneously with the initiation of the protocol.

Serial ultrasonographic controls and $E_{2}$ level measurements were made until 3 follicles $\geq 17 \mathrm{~mm}$ and a serum E2 level $>500 \mathrm{pg} / \mathrm{ml}$ were detected. Choriogonadotropin alpha $250 \mu \mathrm{g}$ S.C. (Ovitrelle ${ }^{\circledR}$; Merck Serono) was administered to induce final follicular maturation. Transvaginal ultrasound-guided oocyte retrieval was performed 35-36 hours after HCG administration, under general anesthesia and only by two gynecologists with at least 5-year experience in the procedure. All patients were implemented a single dose of cefazolin sodium (Sefazol, Mustafa Nevzat Ilaç San., Turkey) $1 \mathrm{gr}$, intramuscularly (I.M.) at the course of OPU procedure and were given doxycycline $100 \mathrm{mg}$ capsule (Tetradox capsule, Fako Ilaç, Turkey) per oral (P.O.) twice daily and methylprednisolone $16 \mathrm{mg}$ capsule (Prednol tablet, Mustafa Nevzat Ilaç San, Turkey) P.O. once daily and continued for 4 days. Luteal support was initiated on the night of oocyte retrieval and continued until the day of pregnancy testing.

Among the included patients, those undergoing OPU for the first time were assigned to group 1 and those who were previously subjected to at least 1 OPU procedures were assigned to group 2. Given the included patients had a complete blood count made before the OPU procedure, another sampling was made immediately before the embryo transfer (ET), which was performed 2 days after the OPU practice. The mean of age, the ratio of agonist/antagonist protocols employed, pre- and postprocedure hemoglobin levels and hemoglobin changes between pre- and post-procedure values, which were expressed as the Delta $\mathrm{Hb}$, were compared between the groups.

Results have been reported as mean \pm SD. Data were analyzed with Statistical Package for the Social Sciences for Windows 22.0 software (SPSS, Chicago, IL., USA). Student's t-test and Mann-Whitney U test were employed to evaluate differences between groups for parametric and non-parametric comparisons, respectively. Categorical data were evaluated by using chi-square test. A two-tailed $\mathrm{p}$ value of $<0.05$ was considered to be statistically significant.

\section{RESULTS}

A total of 553 patient files were assessed for eligibility between June and December 2013 and 53 women were eligible for enrolment and consented to participate in the 
study. Group 1 and 2 consisted of 40 and 13 patients, respectively. Demographic characteristics and agonist/antagonist protocol ratio were comparable between the two groups. Delta hemoglobin value did not significantly differ between the groups $(\mathrm{p}=0.469)$ (Table 1). Additionally, there were no significant difference between the pre- and post-procedure hemoglobin values in the group 1, 2 and the total patient population $(\mathrm{p}=0.792,0.259$ and 0.442 , respectively) (Table 2). No major complication was encountered in neither of the groups. A total of 9 patients $(17 \%)$, including $5(9 \%)$ in group 1 and $4(8 \%)$ in group 2 , experienced minor vaginal bleedings, which were treated with local interventions, such as compression or tamponade, following the procedure.

Table 1: The comparison of the demographic characteristics and the hematologic parameters between the groups.

\begin{tabular}{|lll|l|}
\hline & $\begin{array}{l}\text { Group 1 } \\
(\mathrm{n}=40)\end{array}$ & $\begin{array}{l}\text { Group 2 } \\
(\mathrm{n}=13)\end{array}$ & $\mathrm{p}$ \\
\hline $\begin{array}{l}\text { Age (mean) } \\
\text { (years) }\end{array}$ & $32.5 \pm 8.7$ & $31.9 \pm 3.3$ & $0.469^{*}$ \\
\hline $\begin{array}{l}\text { Agonist/ } \\
\text { Antagonist ratio }\end{array}$ & $18 / 22$ & $6 / 7$ & $0.942^{* *}$ \\
\hline $\begin{array}{l}\text { Minor vaginal } \\
\text { bleeding, }(\%)\end{array}$ & $5(9 \%)$ & $4(8 \%)$ & $0.198^{* *}$ \\
\hline Delta Hb & $-0.04 \pm 0.7$ & $-0.23 \pm 0.7$ & $0.469^{*}$ \\
\hline
\end{tabular}

OPU; oocyte pick-up, Hb; hemoglobin, ET; embryo transfer.

*Mann Whitney-U test, **Chi-square test.

Table 2: The comparison of the pre- and postprocedure values in the groups.

\begin{tabular}{|llll|} 
& $\begin{array}{l}\text { Hb before } \\
\text { OPU }\end{array}$ & $\begin{array}{l}\text { Hb before } \\
\text { ET }\end{array}$ & p* \\
\hline Group 1 & $12.6 \pm 1.3$ & $12.7 \pm 1.2$ & 0.792 \\
\hline Group 2 & $12.2 \pm 1.1$ & $12.7 \pm 1.3$ & 0.259 \\
\hline Total & $12.5 \pm 1.3$ & $12.7 \pm 1.2$ & 0.442 \\
\hline
\end{tabular}

OPU; oocyte pick-up, $\mathrm{Hb}$; hemoglobin, ET; embryo transfer. *student t-test

\section{DISCUSSION}

Though the OPU procedure is performed frequently in the course of ART, the procedure related complications are known to be under-reported and our knowledge on the issue only relies on limited retrospective analyses or case reports. ${ }^{10}$ Severe hemoperitoneum, ${ }^{10,11}$ massive hematuria, $^{12}$ rupture of ovary causing hemorrhagic shock $^{13}$ and uterovaginal fistula formation ${ }^{14}$ have been reported as OPU-related complications. Aragona et al reported the incidence of severe complications and intraperitoneal bleeding, in their 7,098 IVF cycle series, to be $0.08 \%$ and $0.06 \%$, respectively. ${ }^{15}$ However Siristatidis et al reported the rate of severe complications with a higher rate of $0.72 \% .^{1}$ They indicated minor vaginal bleeding to be the most frequent complication, which emerged in the $18.08 \%$ of the patients and was treated easily. In our study, we did not experience any serious hemorrhage complication. We observed procedure-related minor vaginal bleeding in, totally, $17 \%$ of the patients and there existed no significant difference between the groups regarding minor vaginal bleeding ( $9 \%$ and $8 \%$, respectively, $\mathrm{p}=0.198$ ).

Even if not complicated by a major intraperitoneal or pelvic organ bleeding, every OPU procedure is accompanied by a certain amount of blood loss, which is self-limiting and clinically not significant, due to the multiple punctures of the vaginal mucosa, vaginal vault, ovarian surface epithelium and other pelvic structures. A limited number of studies assessing the risk factors for bleeding following transvaginal oocyte retrieval are available in the literature. Zhen et $\mathrm{al}^{9}$ demonstrated in their study, in which they reviewed 10, 251 retrieval cycles over a 4-year period and assessed the risk factors for intraperitoneal bleeding after OPU, that patients with severe intraperitoneal bleeding had a lower BMI and a history of surgery. Additionally, out of 5 patients with severe intraperitoneal bleeding requiring laparotomy or laparoscopy, 4 were reported to have had surgical histories and 1 to have undergone multiple retrieval attempts.

On the other hand, data regarding the blood loss associated with uncomplicated transvaginal oocyte retrieval are quite limited. Ragni et $\mathrm{al}^{5}$ investigated blood loss both biochemically and ultrasonographically at three different times in 150 women undergoing OPU and indicated that at 4-6 hours after OPU, hemoglobin concentration were significantly reduced and pelvic free fluid significantly increased. They finally concluded that the quantity of blood loss following oocyte retrieval is clinically unremarkable in the vast majority of women. To the best to our knowledge, there is no study available regarding the effect of the number of the previous OPU procedures on the amount of bleeding and hematologic parameters and thus, the present is the first in this context. Our study results reveal that the amount of the blood loss does not significantly vary between women who underwent OPU for the first time and for more than 1 time. Even though delta hemoglobin value was greater in women with at least 1 previous OPU than in women undergoing their first OPU, the difference was not statistically significant. Although any history of surgery is known to increase the amount of blood loss in subsequent interventions, we suggest that prior oocyte retrievals do not expose any risk increase.

The limitations of the study include its retrospective design and relatively small number of patients. Even though conducted in a retrospective fashion, the present was performed through the results of CBCs, which were sampled and assayed in line with diverse studies conceptualized and designed as prospective in an unexplained infertility population. In addition, the present is a preliminary study as to provide data firstly regarding the effect of prior oocyte retrievals on blood loss. 
Moreover, excluding all potential factors that could potentially bias the results, including OHSS, PCOS, and high levels of $\mathrm{AMH}$, has rendered the present study more reliable.

\section{CONCLUSIONS}

Based on the results of the present study, we suggest that the number of prior oocyte retrieval procedures do not have any impact on the hematologic parameters obtained after OPU. Thus, women with the history of a prior oocyte retrieval practice may undergo subsequent procedures safely, with regard to blood loss and hemorrhagic complications.

Funding: No funding sources Conflict of interest: None declared

Ethical approval: Not required

\section{REFERENCES}

1. Siristatidis C, Chrelias C, Alexiou A, Kassanos D. Clinical complications after transvaginal oocyte retrieval: a retrospective analysis. J Obstet Gynaecol. 2013;33:64-6.

2. Battaglia C, Regnani G, Giulini S, Madgar L, Genazzani AD, Volpe A. Severe intra-abdominal bleeding after transvaginal oocyte retrieval for IVFET and coagulation factor XI deficiency: a case report. J Assist Reprod Genet. 2001;18:178-81.

3. Bennett SJ, Waterstone JJ, Cheng WC, Parsons J. Complications of transvaginal ultrasound-directed follicle aspiration: a review of 2670 consecutive procedures. J Assist Reprod Genet. 1993;10:72-7.

4. Orvieto R, Ben Rafael Z. Bleeding, severe pelvic infection and ectopic pregnancy following ART. In: Gardner DK, Weissman A, Howles C, Shoam Z, editors. Textbook of assisted reproductive techniques: volume 2: Clinical Perspectives. 4th Edition. London, UK: informa healthcare; 2012:37481.

5. Ragni G, Scarduelli C, Calanna G, Santi G, Benaglia L, Somigliana E. Blood loss during transvaginal oocyte retrieval. Gynecol Obstet Invest. 2009;67:325.

6. Moayeri SE, Coutre SE, Ramirez EJ, Westphal LM. Von Willebrand disease presenting as recurrent hemorrhage after transvaginal oocyte retrieval. Am J Obstet Gynecol. 2007;196:e10-1.

7. El-Shawarby SA, Margara RA, Trew GH, Laffan MA, Lavery SA. Thrombocythemia and hemoperitoneum after transvaginal oocyte retrieval for in vitro fertilization. Fertil Steril. 2004;82:735-7.

8. Ferraretti AP, Goossens V, Kupka M, Bhattacharya S, de Mouzon J, Castilla JA et al. European IVFmonitoring (EIM) consortium for the European Society of Human Reproduction and Embryology (ESHRE). Assisted reproductive technology in Europe, 2009: results generated from European registers by ESHRE: Hum Reprod. 2013;28:2318-31.

9. Zhen X, Qiao J, Ma C, Fan Y, Liu P. Intraperitoneal bleeding following transvaginal oocyte retrieval. Int J Gynaecol Obstet. 2010;108:31-4.

10. Nouri K, Walch K, Promberger R, Kurz C, Tempfer CB, Ott J. Severe haematoperitoneum caused by ovarian bleeding after transvaginal oocyte retrieval: a retrospective analysis and systematic literature review. Reprod Biomed Online. 2014;29:699-707.

11. Kart C, Guven S, Aran T, Dinc H. Life-threatening intraabdominal bleeding after oocyte retrieval successfully managed with angiographic embolization. Fertil Steril. 2011;96:e99-102.

12. Jayakrishnan K, Raman VK, Vijayalakshmi VK, Baheti S, Nambiar D. Massive hematuria with hemodynamic instability-complication of oocyte retrieval. Fertil Steril. 2011;96:e22-4.

13. Bandyopadhyay S, Kay V. A rare case of rupture of ovary causing haemorrhagic shock following uncomplicated oocyte retrieval-a case report. Hum Fertil (Camb). 2010;13:105-6.

14. Mongiu AK, Helfand BT, Kielb SJ. Ureterovaginal fistula formation after oocyte retrieval. Urology. 2009;73:444.e1-3.

15. Aragona C, Mohamed MA, Espinola MS, Linari A, Pecorini F, Micara G et al. Clinical complications after transvaginal oocyte retrieval in 7,098 IVF cycles. Fertil Steril. 2011;95:293-4.

Cite this article as: Özdamar O, Gün I, Ovayolu A, Sofuoğlu K. Does the number of prior oocyte retrieval practices increase the amount of blood loss in subsequent OPU procedures? Int J Reprod Contracept Obstet Gynecol 2016;5:276-9. 